usually exhibit ring forms in the secondary or hereditary types.

From the initial marrow sample the diagnosis of primary sideroblastic anaemia was suggested by the presence of numerous ring-sideroblasts at all stages of erythrocyte maturation. At first the megaloblastic changes were attributed to folate deficiency, in association with primary sideroblastic anaemia. This opinion was revised at an early stage, however, in view of the Schilling test, serum $B_{12}$, and antibody results, which were characteristic of pernicious anaemia. The persistence of sideroblastic change in the marrow despite folate therapy and a response to vitamin $B_{12}$ suggested a concurrent primary sideroblastic anaemia.

The case illustrates the importance of investigating all cases of macrocytic anaemia by iron staining of the marrow together with estimation of the serum $B_{12}$ and folate levels. The possibility that megaloblastosis $\frac{2}{3}$ and sideroblastosis may be due to separate con- $\mathbb{\AA}$ ditions, as in this case, should be considered particu- $c$ larly when associated with a sub-optimal response to $\Rightarrow$ adequate $B_{12}$ replacement therepy.

\section{Acknowledgments}

We wish to thank Dr Eric Jackson for advice and per- $\frac{\bar{\omega}}{-}$ mission to publish details of the case, also Professor M. S. $\mathbb{\Phi}$ Losowsky and Roger Hall for their helpful criticism. Thanks also to Marion Stainthorpe and Mrs Brenda Day for secre-es tarial assistance.

\section{References}

Hall, R. \& Losowsky, M.S. (1966) The distribution of erythroblast iron in sideroblastic anaemias. British Journa of Haematology, 12, 334.

MACGibbon, B.H. \& Mollin, D.L. (1965) Sideroblasticic anaemia in man: observations on seventy cases. British N Journal of Haematology, 11, 59.

\title{
Recurrent intussusception due to a primary colloid carcinoma of the large bowel in a child
}

\author{
M. A. TEHRANI \\ M.D., F.R.C.S. \\ Department of Surgery, Western Infirmary, Glasgow
}

\begin{abstract}
Summary
A case of recurrent colo-colic intussusception due to a primary colloid carcinoma of descending colon with widespread lymphatic involvement in a child, is reported. The unusual mode of presentation and lack of awareness were responsible for a delay of 6 months before the final diagnosis was made. Two years after the operation the patient is well and there is no evidence of clinically detectable secondaries.
\end{abstract}

\section{Introduction}

INTUSSUSCEPTION in adults has a predisposing mechanical cause in more than $50 \%$ of the patients (Donhauser and Kelly, 1950; Goodall, 1963) but in infants and children a demonstrable lesion, in particular a malignant tumour, is uncommon (Strang, 1959; Dennison and Shaker, 1970). The separation between these two groups of patients has been accepted as the adult patients being over 14 years of age (Goodall, 1963; Elebute and Adesola, 1964).

Correspondence: Mr M. A. Tehrani, Department of Surgery, Western Infirmary, Glasgow G11 6NT.
This report concerns a 12-year-old boy with recurrent colo-colic intussusception due to a primaryọ. mucoid carcinoma of the descending colon. The case is of interest because of the rarity of malignant $\mathrm{B}$. tumours of the colon in childhood and adolescencé and the unusual mode of its presentation.

\section{Case report}

A 12-year-old schoolboy was admitted to hospitap with the complaint of abdominal pain. His symptoms began 6 months before admission with intermitten? attacks of colicky lower abdominal pain and disten sion followed by diarrhoea and passage of blood and mucus per rectum. Between these attacks his generat health had been good and he had remained symptom $N$ free. Three weeks before admission, however, some weight loss and diminished appetite were noted byo his parents. He had never vomited but had nausea a times. There were no other symptoms or family? history of consequence.

On examination, he looked anaemic and appeared? tired but had no complaints. He was apyrexial and? normotensive. There were no abnormalities in $\vec{B}$ 
cardiovascular or respiratory systems. The abdomen was not distended and the bowel sounds were normal. The liver and spleen were not enlarged and there was no lymphadenopathy. Rectal examination revealed no mass up to the fingertip but bloodstained faecal material and mucus were observed. The haemoglobin and electrolytes were within normal limits. The initial plain X-rays of the abdomen and chest were of no diagnostic help. Sigmoidoscopy on the same day failed to show an intralumenal lesion, but there was evidence of blood-stained mucus and faeces throughout the rectum and lower sigmoid. Twenty-four hours after admission, while awaiting a barium enema examination, he experienced severe lower abdominal pain followed by the passage of a moderate amount of blood per rectum. The pain, however, persisted-unlike the previous episodes-and he vomited several times. Physical and radiological examination confirmed the presence of large bowel obstruction and an emergency laparotomy was performed. At laparotomy, a colo-colic intussusception of the descending colon was found. After reduction, the cause of the intussusception was found to be an ulcerating tumour encircling three-quarters of the lumen and involving the serosal surface of the bowel (Fig. 1). There were no palpable secondaries in the liver but the mesocolic glands were enlarged and hard and were thought to contain secondary deposits. An emergency radical left hemicolectomy and proximal defunctioning transverse colostomy were performed. Histology showed that the tumour was a colloid carcinoma (Fig. 2) and that all the lymph nodes sectioned contained secondary deposits (Fig. 3). Histological grading of the tumour was that of Dukes's $C$ classi- fication (Dukes, 1940). The patient's post-operative recovery was uneventful and 4 weeks later the colostomy was closed. One week later, however, numerous enlarged nodes appeared in both axillae and a further large node was also palpable over the lower part of the anterior chest wall. Histological examination of the excised nodes from the chest wall and both axillae showed reactive hyperplasia, without evidence of metastatic tumour. He subsequently continued to improve and gained weight. His postoperative immunoglobulin level and lymphocyte transformation test were within normal limits. He received no post-operative radiotherapy or chemotherapy and is now well and symptom-free 2 years after the operation.

\section{Discussion}

The occurrence of intussusception, caused by an organic lesion during infancy and childhood is uncommon. Strang (1959) and Dennison and Shaker (1970) reviewed large series of intussusception over a period of 10 years in infants and children and found a mechanical cause in only $2 \%$ and $3 \%$ respectively and in neither series was a malignant tumour the cause of invagination. In young adults, small bowel tumours are the commonest cause of intussusception (Smith and Gillespie, 1968) but with increase in age, carcinoma becomes an important initiating lesion, particularly in colonic intussusception.

Carcinoma of the large bowel, excluding those occurring in pre-cancerous conditions such as ulcerative colitis and familial polyposis coli, although predominantly a disease of older people may, however, occur at any age. Ahfeld (1880) found a carcinoma of the colon in a stillborn infant with

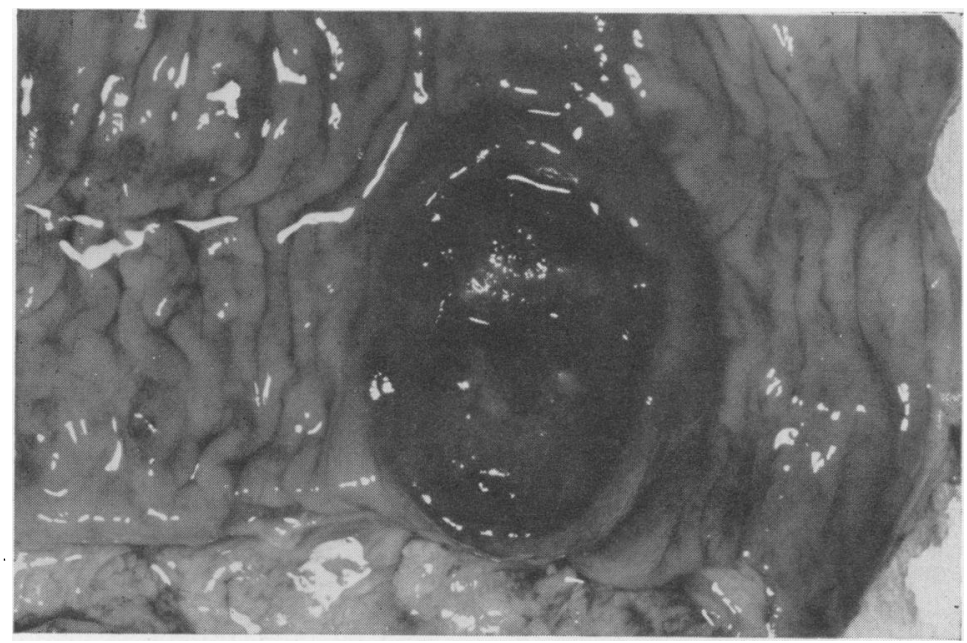

FIG. 1. Gross appearance of the tumour in a part of the excised colonic segment. 


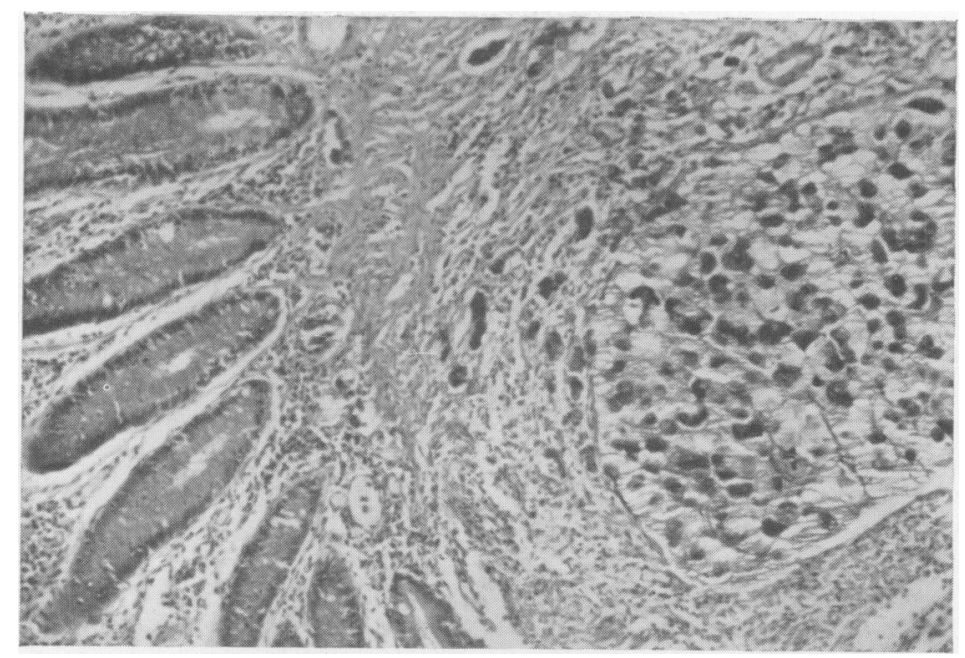

FIG. 2. Microscopic appearance of the tumour showing colloid carcinoma.

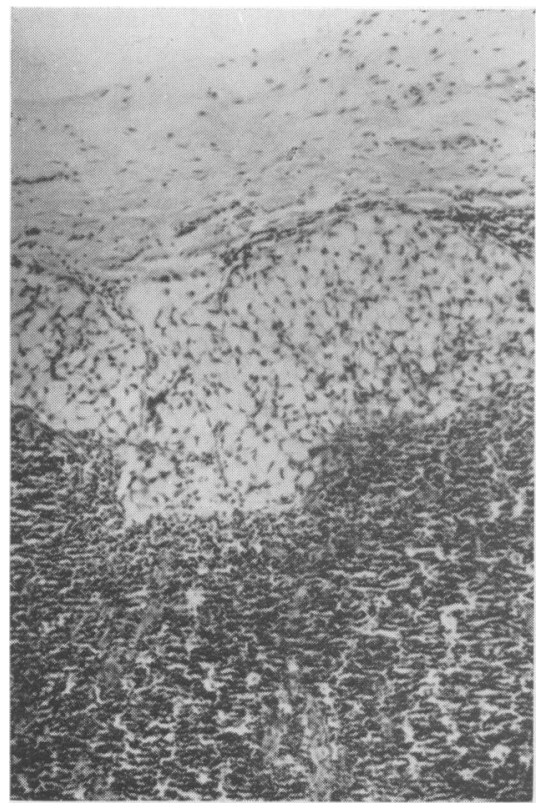

Fig. 3. Mucus-secreting tumour cells in peripheral sinus of the biopsied lymph node.

multiple congenital abnormalities. Since then many individual case reports and collective reviews have been documented (Ritvo, Houghton and McDonald, 1942; Johnston, 1947; Kern and White, 1958; Johnson, Judd and Dahlin, 1959; Pemberton, 1970). The youngest patient reported was that of Kern and White (1958): this was a case of adenocarcinoma of the transverse colon in a 9-month-old girl.

Handy and Goldberg (1956) studied 599 cases of childhood malignancy during the period 1949-1951.

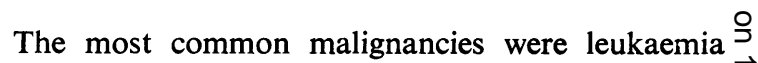
followed by malignancies of the brain, kidney and $\vec{D}$ adrenal. In their series there were twenty-nine cases of digestive system tumours $(4.8 \%)$, an incidence of 0.6 cases for every 100,000 children under 15 years $\mathscr{\theta}$ age. Alaghemand (1962) collected and analys ninety-three cases of colo-rectal malignancy 两. children 15 years old and under, and found that the large bowel was more frequently affected by cancer in childhood than any other part of the alimentary tract. Similar observation had been made by $\propto$ Bonelli (1947) who reported an incidence of $91.5 \% \overrightarrow{\overrightarrow{\vec{O}}}$ of colo-rectal involvement in a series of 142 cases of $\frac{3}{3}$ carcinoma of the small and large bowel in infants and children.

Review of the literature shows that the most common presenting symptom is recurrent abdominal 3 . pain; appendicitis and/or mesenteric adenitis being the initial erroneous diagnosis. More frequent use of other diagnostic method such as plain radiograph, $\delta$ sigmoidoscopy and barium studies, therefore, should $₹$ be made when dealing with recurrent abdominal $\frac{9}{5}$ pain in children and adolescents (Pemberton, 1970). $>$ Unfortunately, there is an invariable delay in making a diagnosis, as abdominal pain is a very common $N$ complaint in this age group and the early appearance $\%$ of the classical triad of change in bowel habit, passage of blood and mucus per rectum, and ob- $\underset{\omega}{N}$ structive symptoms is exceptional (O'Brien, 1967). 으 According to the literature, prognosis of colonice carcinoma in young patients is poor and often hope- $\Phi$ less. In follow-up studies of fifty-one cases in Alag- $\stackrel{?}{+}$ hemand's series (1962), 5-year survival was reported 꿍 in only one case. Factors contributing to the generally $\frac{\vec{D}}{\mathrm{D}}$

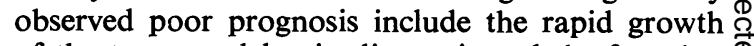
of the tumour, delay in diagnosis and the fact that $\stackrel{\mathbb{Q}}{\Omega}$ 
over $50 \%$ of these tumours are colloid carcinoma as compared with $5 \%$ incidence in adults (Williams, 1955; O'Brien, 1967). The colloid material seems to facilitate the early and extensive spread of the tumour cells through adjacent tissue plane (Chappell, 1959). Early diagnosis and treatment therefore becomes imperative. The recommended treatment is primary resection and anastomosis even in the presence of large bowel obstruction, as delay caused by two stage procedures may result in fur ther metastatic spread.

At laparotomy an important prognostic feature is the presence or absence of the lymphatic spread. Among those with secondary lymphatic involvement, few long-term survivors have been reported (Johnson et al., 1959). In both patients described by Middelkamp and Haffner (1963) and Pemberton (1970) who have survived so long, no metastases were found in the regional lymph nodes which were examined.

The patient in this report had obviously suffered from frequent episodes of incomplete colo-colic intussusception of short duration with spontaneous reduction during which time he had remained symptom-free. Presentation as recurrent intussusception has not previously been described although, in late childhood, established intussusception is often co-existent with colonic tumours (Alaghemand, 1962).

In this case, the unusual mode of presentation associated with lack of awareness were responsible for a delay of 6 months before the final diagnosis was made.

It is concluded that the clinician should not be influenced by the age of the patient and, although uncommon, malignant tumours of the large bowel warrant consideration in differential diagnosis of recurrent abdominal pain in childhood and adolescence.

\section{Acknowledgment}

I would like to thank Mr D. G. Young of the Royal
Hospital for Sick Children, Glasgow, for his helpful criticism during preparation of this paper.

\section{References}

Ahfeld, F. (1880) Zur Casuistik der congenitalen Neoplasmen. Archiv für Gynaekologie, 16, 135.

Alaghemand, A. (1962) Carcinoma of the colon and rectum in children. American Surgeon, 28, 784.

BONELLI, W.R. (1947) Malignant tumors of the small and large intestines in infants and children. Clinical Proceedings of the Children's Hospital, Washington, 3, 151.

Chappell, F.W. (1959) Carcinoma of the colon in young people. American Surgeon, 25, 449.

DenNison, W.M. \& Shaker, M. (1970) Intussusception in infancy and childhood. British Journal of Surgery, 57, 679.

Donhauser, J.L. \& Kelly, B.C. (1950) Intussusception in adult. American Journal of Surgery, 79, 673.

DukEs, C.E. (1940) Cancer of the rectum; an analysis of 1000 cases. Journal of Pathology and Bacteriology, 50, 527.

Elebute, E.A. \& Adesola, A.O. (1964) Intussusception in Western Nigeria. British Journal of Surgery, 51, 440.

GoodAll, P. (1963) Intussusception in adults complicating specific inflammatory diseases of the intestine. Gut, 4, 132.

HANDY, V.H. \& GoldBerG, I.D. (1956) The occurrence of malignancies in children. New York State Journal of Medicine, 56, 258.

Johnson, J.W., JudD, E.S. \& DAHLIN, D.C. (1959) Malignant neoplasms of the colon and rectum in young persons. Archives of Surgery, 79, 365.

Johnston, J.H., JR (1947) Carcinoma of the colon in childhood and adolescence. American Journal of Surgery, 73, 703.

KeRN, W.H. \& White, W.C. (1958) Adenocarcinoma of the colon in a 9-month-old infant. Cancer, 11, 855.

Middelkamp, J.N. \& HaffNer, H. (1963) Carcinoma of the colon in children. Pediatrics. Springfield, Illinois. 32, 558.

O'BRIEN, S.E. (1967) Carcinoma of the colon in childhood and adolescence. Canadian Medical Association Journal, 96, 1217.

Pemberton, M. (1970) Carcinoma of the large intestine with survival in a child of nine and in his father. British Journal of Surgery, 57, 841 .

Ritvo, M., Houghton, J.D. \& McDonald, E.J. (1942) Cancer in childhood. Radiology, 39, 278.

Smith, I.S. \& Gillespie, G. (1968) Adult intussusception in Glasgow. British Journal of Surgery, 55, 925.

Strang, R.P. (1959) Intussusception in infancy and childhood. A review of 400 cases. British Journal of Surgery, 46. 484.

Williams, C., JR (1954) Carcinoma of the colon in childhood. Annals of Surgery, 139, 816. 